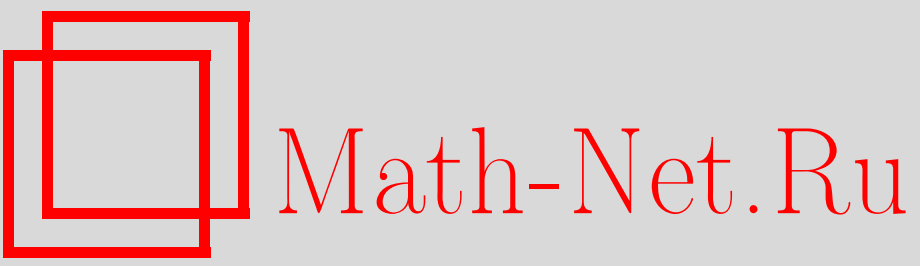

И. Г. Корепанов, Евклидовы 4-симплексы и инварианты четырехмерных многообразий. II. Алгебраический комплекс и перестройки $2 \leftrightarrow 4, T M \Phi, 2002$, том 133, номер 1, 24-35

DOI: https://doi.org/10.4213/tmf378

Использование Общероссийского математического портала Math-Net.Ru подразумевает, что вы прочитали и согласны с пользовательским соглашением

http: //www . mathnet.ru/rus/agreement

Параметры загрузки :

IP : 54.197 .217 .227

26 апреля 2023 г., 13:26:12 


\section{ЕВКЛИДОВЫ 4-СИМПЛЕКСЫ И ИНВАРИАНТЫ ЧЕТЫРЕХМЕРНЫХ МНОГООБРАЗИЙ. II. АЛГЕБРАИЧЕСКИЙ КОМПЛЕКС И ПЕРЕСТРОЙКИ $2 \leftrightarrow 4$}

Приводятся диаграммы линейных отображений векторных пространств с фиксированными базисами. Каждый член диаграммы есть линейное пространство диффференциалов метрических величин, приписанных элементам симплициального комплекса триангуляции многообразия. Если диаграмма оказывается ациклическим комплексом, то из его кручения можно построить инвариант многообразия. Это демонстрируется вначале на трехмерных многообразиях, затем часть аналогичной работы, связанная с перестройками $2 \leftrightarrow 4$, проводится для четырехмерных многообразий.

Ключевые слова: кусочно-линейные многообразия, инварианты многообразий, движения Пахнера, дифференциальные тождества для евклидовых симплексов, ациклические комплексы.

\section{1. ВВЕДЕНИЕ}

Настоящая работа является второй в серии статей (первой была работа [1]), цель которых - построение инвариантов четырехмерных кусочно-линейных многообразий, аналогичных инвариантам трехмерных многообразий, построенных в работах [2] и [3]. В статье [1] рассматривались только перестройки (движения Пахнера) типа $3 \rightarrow 3$, когда три 4-симплекса из триангуляции многообразия заменяются на другие три симплекса с той же общей границей. Ниже мы будем часто ссылаться на формулы, теоремы и пр. из этой статьи, снабжая их римской цифрой I (а саму статью [1] называть работой I). Например: "Выражение для инварианта перестроек $3 \rightarrow 3$ приведено в формуле (I.23)".

Оказьвается, что дальнейшее построение наших инвариантов естественно вести на алгебраическом языке ациклических комплексов. Более того, уже в трехмерном случае одна из конструкций, использованных в работах [2] и [3], естественно интерпретируется как построение кручения подходящих ациклических комплексов.

В разделе 2 мы рассмотрим трехмерный случай с этих новых позиций. Помимо самостоятельного интереса, его изучение подготавливает построение и исследование алгебраического комплекса для четырехмерных многообразий. Обший вид этого комплекса и

\footnotetext{
* Южно-Уральский государственный университет, Челябинск, Россия. E-mail: kig@susu.ac.ru
} 
план работы в четырехмерном случае даны в разделе 3 . Часть этого плана реализуется в настоящей статье, а часть - в планируемой статье III этой серии.

В разделе 4 настоящей статьи будут введены девиации ребер, составляюшие одно из линейных пространств в нашем комплексе. В разделе 5 мы частично оправдываем название комплекс, доказывая теорему о том, что образ одного из отображений лежит в ядре следующего (полностью эту работу для случая четырех измерений намечено провести в работе III). В разделе 6 объясняется связь девиаций ребер с перестройками $2 \leftrightarrow 4$, т.е. с переходом от кластера из двух 4-симплексов к кластеру из четырех и наоборот.

В заключительном разделе 7 мы подведем итоги статьи I и настояшей статьи и наметим цели следующей работы этой серии.

\section{2. ВОЗВРАШЕНИЕ К ТРЕХМЕРНОМУ СЛУЧАЮ: ИНВАРИАНТ $I$ КАК КРУЧЕНИЕ АЛГЕБРАИЧЕСКОГО КОМПЛЕКСА}

Напомним, что в работах [2] и [3] рассматриваются ориентируемые трехмерные многообразия, представленные в виде симплициального комплекса (или предкомплекса, в котором симплекс может входить несколько раз в границу симплекса большей размерности). Каждому ребру приписывается евклидова длина, а каждой 3-клетке (тетраэдру) - знак “+" или "_" таким способом, что удовлетворяется следуюшее условие нулевой кривизны: алгебраическая сумма двугранных углов, примыкаюших к каждому ребру, равна нулю по модулю $2 \pi$. Здесь каждый двугранный угол определяется длинами ребер соответствующего тетраэдра и берется со знаком, сопоставленным этому тетраэдру. Далее рассматриваются бесконечно малые вариации длин ребер и линейно зависяшие от них бесконечно малые угль дефекта при ребрах. Выражаюшая эти зависимости матрица $A$ играет ключевую роль в итоговой формуле для инвариантов многообразий.

Матришу $A$ можно рассматривать как линейное отображение одного векторного пространства с фиксированным базисом в другое аналогичное. Оказывается, $A$ естественным образом объединяется с четырьмя другими линейными отображениями пространств с заданными базисами в точную последовательность, или ациклический комплекс, который можно записать следующим образом:

$$
0 \leftarrow(\cdots) \stackrel{B^{\mathrm{T}}}{\leftarrow}(d \omega) \stackrel{A}{\leftarrow}(d l) \stackrel{B}{\leftarrow}(d x \text { и } d g) \leftarrow 0 .
$$

Здесь $(d x)$ - совокупность дифференциалов координат вершин комплекса, взятых с точностью до движений евклидова пространства (например, в разделе 3 работы [3] это $\varphi$, $\sigma, s$ и $\alpha) ;(d g)$ - дифференшиалы непрерывных параметров, от которых может зависеть представление фундаментальной группы многообразия в $E_{3}$ (случай бесконечной фундаментальной группы будет рассмотрен ниже); $(d l)$ - столбец дифференциалов длин ребер; $(d \omega)$ - столбец дифференциалов углов дефекта. Напомним, что верхний индекс Т означает матричное транспонирование и $A=A^{\mathrm{T}}$. Левая половина последовательности (1) получается из правой транспонированием всех матриц, поэтому точность в членах $(\cdots)$ и $(d \omega)$ получается из точности в симметричных им членах. Мы можем не 
выяснять геометрического смысла величин, обозначенных $(\cdots),-$ из точности последовательности будет следовать, что это просто элементы пространства $\operatorname{Im} B^{\mathrm{T}}$ - образа отображения $B^{\mathrm{T}}$.

Нам понадобится следующая лемма.

ЛЕмма 1. Если длины ребер комплекса и знаки тетраэдров выбраны так, что все угль дефекта равны нулю, то вериины универсальной накрывающей комплекса можсно поместить в евклидово пространство $\mathbb{R}^{3}$ так, что длины совпадут с расстояниями между вершинами, а знаки тетраэдров - с ориентациями их образов в $\mathbb{R}^{3}$. Это делается однозначно с точностью до движсений пространства $\mathbb{R}^{3}$.

ДокАЗАТЕЛЬСтво. Выберем в универсальной накрывающей один тетраэдр (назовем его первылм) и свяжем с ним евклидову систему координат. Ее можно естественно продолжить на любой смежный тетраэдр, т.е. имеющий общую грань с первым (напомним, что каждый отдельный тетраэдр всегда вкладывается в $\mathbb{R}^{3}$ ). Продолжая эту процедуру, можно ввести координаты в любом тетраэдре, соединив его с первым последовательностью тетраэдров, каждый из которых смежен с двумя соседними. Геометрически представим эту последовательность как ломаную линию, начинаюшуюся в барицентре первого тетраэдра и кончающуюся в барицентре последнего, каждый прямолинейный отрезок которой соединяет барицентры двух смежных тетраэдров.

Докажем, что если есть две таких ломаных линии с общими началом и концом, то они приводят к одной и той же системе координат в последнем тетраэдре. Допустим, что это не так. Тогда при обходе по замкнутому пути с началом и концом в последнем тетраэдре мы получаем в нем измененную систему координат. Этот путь может быть стянут (ведь мы находимся в универсальной накрывающей) в барицентр последнего тетраэдра, причем можно считать, что при этом стягивании он пересекает только ребра (не вершины) комплекса.

Из того, что угол дефекта вокруг каждого ребра равен нулю, следует, что евклидовы координаты, заданные в одном из тетраэдров, содержащих это ребро, однозначно и непротиворечиво распространяются на все тетраэдры, содержащие это ребро. Поэтому матрица монодромии, описываюшая изменение системы координат при обходе по нашему контуру, не меняется, когда мы его протаскиваем через ребро. В конце концов мы получим путь, состоящий из единственной точки, и две системы координат. Это противоречие завершает доказательство леммы.

Достаточно строго мы определим члены последовательности (1) и докажем ее точность только для многообразий $M$ с конечной фундаментальной группой $\pi_{1}(M)$, и лишь на примере покажем, чего можно ожидать в случае бесконечной групшы. Напомним [3], что наш инвариант зависит также от представления $f: \pi_{1}(M) \rightarrow E_{3}$. Начнем со случая, которому посвяшена работа [3]: $M=L(p, q)$ с нетривиальным представлением группы $\pi_{1}(L(p, q))=\mathbb{Z}_{p}$ в $E^{3}$ врашениями вокруг оси $z$.

При взятии универсальной накрываюшей каждая вершина триангуляции $L(p, q)$ размножается в $p$ раз. Получившиеся $p$ точек мы располагаем в вершинах правильного $p$-угольника, так чтобы они переходили друг в друга при врашениях вокруг оси $z$ на уг- 
лы, кратные $2 \pi / p$. Но в отличие от работы [3] будем рассматривать произвольную триангуляцию - с любым числом вершин. Пусть число вершин равно $m$, тогда их взаимное расположение определяется, во-первых, $m$ расстояниями $\rho_{1}, \ldots, \rho_{m}$ от них до оси $z$ и, во-вторых, $(m-1)$ разностями значений каждой из двух остальных цилиндрических координат, например, $\left(\varphi_{2}-\varphi_{1}\right), \ldots,\left(\varphi_{m}-\varphi_{1}\right)$ и $\left(z_{2}-z_{1}\right), \ldots,\left(z_{m}-z_{1}\right)$. Опыт работы [3] (см. формулу (3.16)) показывает, что совокупность дифференциалов $d x$ следует выбрать в виде

$$
(d x)=\left(\rho_{1} d \rho_{1}, \ldots, \rho_{m} d \rho_{m} ; d\left(\varphi_{2}-\varphi_{1}\right), \ldots, d\left(\varphi_{m}-\varphi_{1}\right) ; d\left(z_{2}-z_{1}\right), \ldots, d\left(z_{m}-z_{1}\right)\right)
$$

Что касается дифференциалов $(d g)$, то они в данном случае отсутствуют: представление конечной группы не деформируется непрерывно в неэквивалентое.

Отображение $B$ определяет изменения длин всех ребер комплекса для данных $(d x)$. Инъективность его для $\rho, \varphi$ и $z$, находящихся в общем положении, очевидна: соответствие между $\rho_{i}, \varphi_{i}-\varphi_{1}, z_{i}-z_{1}$ и длинами ребер комплекса, даюшими нулевые углы дефекта, локально взаимно однозначно (это следует из леммы 1), и поэтому якобиан отображения В почти всюду ненулевой. Инъективность $B$ означает точность последовательности (1) в члене $(d x)$.

Остается доказать точность в члене $(d l)$. То, что $\operatorname{Im} B \subset \operatorname{Ker} A$, очевидно: если дифференциалы длин порождены сдвигами вершин в евклидовом пространстве $\mathbb{R}^{3}$, то углы дефекта равны нулю. Докажем, что $\operatorname{Ker} A \subset \operatorname{Im} B$. Это означает, что если бесконечно малые углы дефекта равны нулю, то наш комплекс - универсальную накрывающую триангуляции $L(p, q)$ - можно поместить в евклидово пространство $\mathbb{R}^{3}$. Но это - инфинитезимальная версия все той же леммы 1 (с тем же доказательством).

На этом доказательство точности последовательности (1) для $L(p, q)$ закончено. Обобшим этот результат для всех (связных замкнутых ориентируемых) многообразий $M$ с конечной фундаментальной группой $\pi_{1}(M)$. Единственное существенное отличие от случая $L(p, q)$ может состоять в выборе переменных $(d x)$. Как видно из работ [2], [3], они должны обладать следующим свойством: при добавлении новой вершины $E$ (в результате перестройки $1 \rightarrow 4)$ форма $\wedge d x$ должна умножаться на $d x_{E} \wedge d y_{E} \wedge d z_{E}-$ форму евклидова объема. Напомним, что наш инвариант соответствует паре $(M, f)$, где $f: \pi_{1}(M) \rightarrow E_{3}$. Возможны три ситуации в зависимости от образа $\operatorname{Im} f$ группы $\pi_{1}(M)$ в $E_{3}$.

1. $\operatorname{Im} f=\{e\}$. Этот случай рассмотрен в работе [2].

2. $\operatorname{Im} f$ состоит из вращений вокруг только одной оси. В этом случае справедливы рассуждения, приведенные выше для $L(p, q)$. В частности, $(d x)$ нужно выбирать по формуле (2).

3. $\operatorname{Im} f$ содержит вращения вокруг как минимум двух (непараллельных) осей. Наличие таких осей фиксирует систему координат в $\mathbb{R}^{3}$, и совокупность $(d x)$ должна состоять из всех дифференциалов евклидовых координат вершин комплекса:

$$
(d x)=\left(d x_{1}, d y_{1}, d z_{1}, \ldots, d x_{m}, d y_{m}, d z_{m}\right)
$$


Построив ациклический комплекс (1) для многообразия $M$ с конечной группой $\pi_{1}(M)$, вычислим его кручение. Как и в работах [2], [3], выберем максимальное подмножество $\mathcal{C}$ в множестве ребер триангуляции $M$ такое, что квадратная матрица $\left.A\right|_{\mathcal{C}}$ (получающаяся из $A$ удалением строк и столбцов, соответствуюших ребрам из $\overline{\mathcal{C}}$ - дополнения $\mathcal{C}$ ) невырожденна. Нам еше понадобится матрица $\left.B\right|_{\overline{\mathcal{C}}}$, получающаяся удалением из матрицы $B$ строк, соответствующих ребрам из $\mathcal{C}$. Согласно обычным формулам [4], кручение $\tau$ есть

$$
\tau=\frac{\left(\left.\operatorname{det} B\right|_{\overline{\mathcal{C}}}\right)^{2}}{\left.\operatorname{det} A\right|_{\mathcal{C}}}
$$

Как известно, $\tau$ не зависит от выбора $\mathcal{C}$.

В стиле работ [2], [3] можно записать:

$$
\left.\operatorname{det} B\right|_{\overline{\mathcal{C}}}=\frac{\bigwedge_{\overline{\mathcal{C}}} d l}{\bigwedge d x}
$$

после чего их перевод на наш новый язык не представляет труда. Сформулируем результат в виде следующей теоремы.

Tеорема 1. Величина

$$
\frac{\tau \prod_{\text {по всем ребрам }} l^{2}}{\prod_{\text {по всем тетраэдрам }} 6 V}
$$

является инвариантом трехмерного связного замкнутого ориентируемого многообразия с конечной фундаментальной группой.

Разумеется, (5) есть квадрат инварианта $I$ из [2] или $I_{k}$ из [3] в тех случаях, которые рассматривались в этих работах.

ДоКАЗАТЕЛЬСТво теоремы 1 состоит в разборе того, что́ происходит с выражением (5) при перестройках $2 \leftrightarrow 3$ и $1 \leftrightarrow 4$, а это делается совершенно так же, как в работах [2] и [3]. Теорема доказана.

Покажем теперь, чего можно ожидать в случае бесконечной группы $\pi_{1}(M)$ на примере многообразия $M$ с $\pi_{1}(M)=\mathbb{Z}$ (например, $M=S^{1} \times S^{2}$ ). В общем случае образующая групшы $\pi_{1}(M)$ переводится гомоморфизмом $f$ в поворот вокруг некоторой оси пусть это будет ось $z$ - на угол $\alpha$ и сдвиг вдоль этой же оси на расстояние $a$. Рассуждения, аналогичные приведенным выше, показывают, что точную последовательность (1) мы получим, добавив в качестве $(d g)$ дифференциалы $d \alpha$ и $d a$ к таким же $(d x)$, как в формуле (2). После этого кручение и инвариант вычисляются по прежним формулам (4) и (5). Мы не останавливаемся на этом подробнее и лишь выдвигаем гипотезу, что для всех $M$ можно так выбрать $(d x)$ и $(d g)$, что (1) будет точной последовательностью. 


\section{3. ЧЕТЫРЕХМЕРНЫЙ СЛУЧАЙ: ОБШИЙ ВИД АЛГЕБРАИЧЕСКОГО КОМПЛЕКСА}

Выпишем последовательность линейных отображений пространств с фиксированными базисами - кандидата на роль ациклического комплекса, аналогичного (1), в четырехмерном случае. Точнее, мы выпишем две сопряженные последовательности матрицы, задаюшие отображения в одной из них, будут получаться из матриц другой транспонированием. Это позволит при исследовании точности в различных членах пользоваться той из последовательностей, которая удобнее в данном случае, и, более того, обойти вопрос о геометрическом смысле некоторых линейных пространств, участвующих в последовательности, если смысл соответствующего участка другой последовательности ясен и этот участок поддается изучению. Такие линейные пространства мы будем обозначать многоточием $(\cdots)$, используя это обозначение для разных линейных пространств, которые (пока) не используются в наших рассуждениях.

Определение некоторых линейных пространств, входящих в первую из последовательностей, будет дано в следующих разделах настоящей статьи и в следующих работах этой серии. Тем не менее мы считаем целесообразным выписать их уже сейчас, потому что они будут определять план нашей дальнейшей работы и взаимосвязь ее отдельных частей:

$$
\begin{gathered}
0 \leftarrow(\cdots) \leftarrow\left(d \Omega_{a}\right) \stackrel{\left(\partial \Omega_{a} / \partial S_{i}\right)}{\longleftarrow}\left(d S_{i}\right) \leftarrow\left(d \vec{v}_{a}\right) \leftarrow(d \sigma) \leftarrow 0, \\
0 \rightarrow(d x \text { и } d g) \rightarrow\left(d L_{a}\right) \stackrel{\left(\partial \omega_{i} / \partial L_{a}\right)}{\longrightarrow}\left(d \omega_{i}\right) \rightarrow(\cdots) \rightarrow(\cdots) \rightarrow 0 .
\end{gathered}
$$

Объяснения начнем с последовательности (7). Ее левая часть до $\left(d \omega_{i}\right)$ включительно является прямым аналогом правой части диаграммы (1). Напомним только, что $L_{a}-$ это квадрат длины $a$-го ребра, а $\omega_{i}$ - угол дефекта вокруг $i$-й двумерной грани.

Связуюшим звеном между (7) и (6) служит формула (I.16), утверждаюшая взаимную сопряженность матриц $\left(\partial \Omega_{a} / \partial S_{i}\right)$ и $\left(\partial \omega_{i} / \partial L_{a}\right)$, где $S_{i}$ - плошадь $i$-й двумерной грани, a $\Omega_{i}$ - угол дефекта вокруг $a$-го ребра, определенный формулой (I.13). Величины $d \vec{v}_{a}-$ мы назовем их девиациями ребер - вводятся в разделе 4 вместе с отображением $\left(d \vec{v}_{a}\right) \rightarrow$ $\left(d S_{i}\right)$. Что касается величин $d \sigma-$ девиаций вериин, то их определение мы оставим до следуюшей работы этой серии.

По-видимому, есть достаточно много интересных многообразий, для которых последовательности (6) и (7) являются ациклическими комплексами, и, значит, можно надеяться получить инварианты четырехмерных многообразий из их кручения аналогично формуле (5). В этой статье мы сосредоточимся на том, что можно получить из участка $\left(d \Omega_{a}\right) \leftarrow\left(d S_{i}\right) \leftarrow\left(d \vec{v}_{a}\right)$ последовательности $(6)$. Оказывается, в известном смысле он отвечает за перестройки $2 \leftrightarrow 4$.

\section{4. ДЕВИАЦИИ РЕБЕР И ОПРЕДЕЛЯЕМЫЕ ИМИ ДИФФЕРЕНЦИАЛЫ ПЛОШАДЕЙ}

В этом разделе мы введем понятие девиаций ребер и объясним, как они порождают дифференциалы площадей двумерных граней, иными словами, как строится матрища $\left(\partial S_{i} / \partial \vec{v}_{a}\right)$, задающая отображение $\left(d \vec{v}_{a}\right) \rightarrow\left(d S_{i}\right)$ в последовательности $(6)$. 
Пусть $A B$ - некоторое ребро нашего комплекса. Напомним, что в разделе 3 работы I мы отобразили все клетки универсальной накрывающей комплекса в евклидово пространство $\mathbb{R}^{4}$. Используя поднятие ребра $A B$ и примыкаюших к нему 4-симплексов в универсальную накрывающую, мы можем считать, что это ребро и соседние с ним элементы комплекса помешены в $\mathbb{R}^{4}$.

Девиацией ребра $A B$ будем называть бесконечно малый вектор $d \vec{v}_{A B}$, перпендикулярный $A B$, начало которого находится в середине ребра.

Площадь любого евклидова треугольника равна учетверенной плошади треугольника с вершинами в серединах его сторон. Если девиации сторон треугольника $A B C$ суть $d \vec{v}_{A B}, d \vec{v}_{A C}$ и $d \vec{v}_{B C}$, то положим по определению порождаемый ими дифференциал $d S_{A B C}$ равным (см. рис. 1$)$

$$
d S_{A B C}=4 S_{A^{\prime} B^{\prime} C^{\prime}}-S_{A B C} .
$$

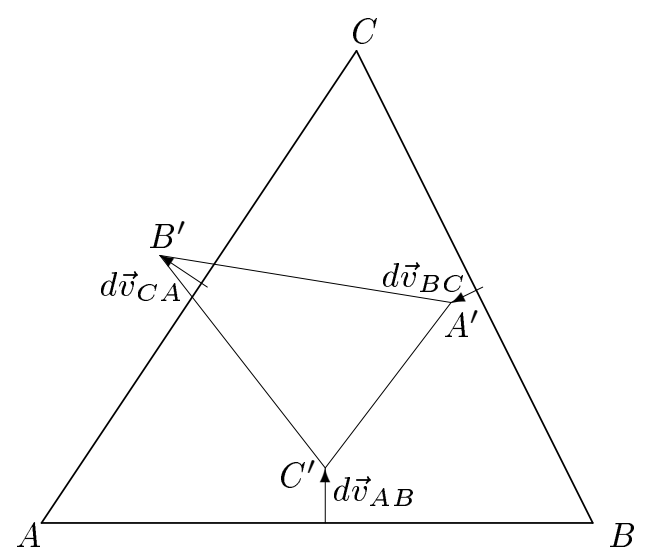

Рис. 1. Иллюстрация к формуле (8).

Пусть имеется 4-симплекс $A B C D E$, лежаший в евклидовом пространстве $\mathbb{R}^{4}$ с координатами $(x, y, z, t)$. Будем считать, что вершины $A$ и $B$ лежат на оси $t$. Рассмотрим ситуацию, когда только ребро $A B$ имеет ненулевую девиацию $d \vec{v}_{A B}=d \vec{v}=$ $\left(d v_{x}, d v_{y}, d v_{z}, 0\right)$, и выведем формулу, связываюшую $d v_{x} \wedge d v_{y} \wedge d v_{z}$ и $d S_{A B C} \wedge$ $d S_{A B D} \wedge d S_{A B E}$.

Спроецируем симплекс $A B C D E$ вдоль оси $t$ на трехмерное пространство, обозначив проекции точек $A, \ldots, E$ теми же буквами (рис.2). Точка $C_{1 / 2}$ на рис. 2 - общая проекция середин отрезков $A C$ и $B C$; аналогично $D_{1 / 2}$ - проекция середин отрезков $A D$ и $B D$, а $E_{1 / 2}$ - отрезков $A E$ и $B E ; K$ - проекция точки, отстоящей от середины ребра $A B$ на девиацию $d \vec{v}$.

Отвлекаясь временно от четырехмерного происхождения рис. 2 и пользуясь несложной тригонометрией (ср. работа [2], формулы (31), (32)), можно записать:

$$
\left|d v_{x} \wedge d v_{y} \wedge d v_{z}\right|=\left|\frac{l_{A C_{1 / 2}} d l_{K C_{1 / 2}} \wedge l_{A D_{1 / 2}} d l_{K D_{1 / 2}} \wedge l_{A E_{1 / 2}} d l_{K E_{1 / 2}}}{6 V_{A C_{1 / 2} D_{1 / 2} E_{1 / 2}}}\right|
$$




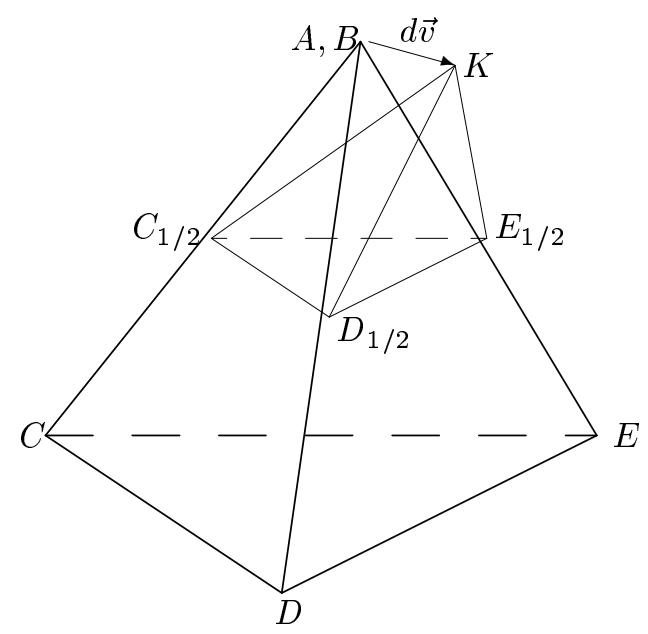

Рис. 2. Трехмерная проекция 4-симплекса $A B C D E$ и девиации ребра $A B$.

Здесь, разумеется, $d l_{K C_{1 / 2}}=l_{K C_{1 / 2}}-l_{A C_{1 / 2}}$ и т.д.; сама буква $l$ обозначает длину соответствуюшего отрезка в трехмерном пространстве, а $V$ - трехмерный объем.

Теперь вспомним о четвертом измерении. Обозначим длину отрезка $A B$ через $h$. Из того, что этот отрезок ортогонален изображенной на рис. 2 трехмерной проекции, следует, что

$$
\left|6 V_{A C_{1 / 2} D_{1 / 2} E_{1 / 2}} \cdot h\right|=\left|3 V_{A B C D E}\right|
$$

(как обычно, ставим модули, чтобы не заботиться об ориентации);

$$
l_{A C_{1 / 2}} \cdot h=S_{A B C}, \quad d l_{K C_{1 / 2}} \cdot h=d S_{A B C}
$$

еше две пары формул типа (9) получаются заменами $C \rightarrow D$ и $C \rightarrow E$.

В результате получаем

$$
L_{A B}^{5 / 2}\left|d v_{x} \wedge d v_{y} \wedge d v_{z}\right|=\left|\frac{d\left(S_{A B C}^{2}\right) \wedge d\left(S_{A B D}^{2}\right) \wedge d\left(S_{A B E}^{2}\right)}{24 V_{A B C D E}}\right|
$$

где мы вернулись к обозначению $L_{A B}=h^{2}$ для квадрата длины отрезка, введенному в работе I.

\section{5. ДЕВИАЦИИ РЕБЕР ДАЮТ НУЛЕВЫЕ УГЛЫ ДЕФЕКТА $d \Omega$}

Следующая теорема дает "половину" точности последовательности (6) в члене $\left(d S_{i}\right)$.

ТЕОрема 2. Для последовательности (6) $\operatorname{Im}\left(\partial S_{i} / \partial \vec{v}_{a}\right) \subset \operatorname{Ker}\left(\partial \Omega_{a} / \partial S_{i}\right)$. 
ДОКАЗАТЕЛЬСТвО составляет всю оставшуюся часть этого пункта.

Совокупность всех дифференциалов $d S_{i}$ плошадей двумерных граней комплекса мы представим в виде вектора-столбца $\left(d S_{i}\right)$. Рассмотрим сначала, какие $d S_{i}$ возникают из девиаций одного ребра $A B$, обшего ровно четырем 4-симплексам $A B C D E=\widehat{F}$, $A B C F D=-\widehat{E}, A B C E F=\widehat{D}$ и $A B D F E=-\widehat{C}$ (записанные в таком виде, эти симплексы имеют согласованную ориентацию). Так как $A B$ является обшим также для ровно четырех двумерных граней, вектор $\left(d S_{i}\right)$ может иметь в данном случае четыре ненулевых компоненты. Оказывается, что они связаны линейной зависимостью

$$
V_{-\widehat{C}} d\left(S_{A B C}^{2}\right)+V_{\widehat{D}} d\left(S_{A B D}^{2}\right)+V_{-\widehat{E}} d\left(S_{A B E}^{2}\right)+V_{\widehat{F}} d\left(S_{A B F}^{2}\right)=0 .
$$

В самом деле, из формулы (10) видно, что выражение

$$
\left|d\left(S_{A B C}^{2}\right) \wedge d\left(S_{A B D}^{2}\right) \wedge d\left(S_{A B E}^{2}\right) / V_{\widehat{F}}\right|
$$

не меняется при перестановках букв $C, D, E, F$. Нетрудно проверить, что именно линейная зависимость (11) обеспечивает эту неизменность. Точнее, из неизменности выражения (12) слагаемые в уравнении (11) устанавливаются с точностью до знаков, которые легко найти, например, рассматривая предельные случаи, когда одна из точек $D, E$ или $F$ расположена очень близко к точке $C$ : если $D \rightarrow C$, то дифференциалы $d S_{A B C}$ и $d S_{A B D}$ должны быть одинаковыми.

Докажем, что уравнение (11) эквивалентно условию $d \Omega_{A B}=0$, где $d \Omega_{A B}-$ угол дефекта вокруг ребра $A B$ - рассматривается как функция $d S_{A B C}, d S_{A B D}$, $d S_{A B E}$ и $d S_{A B F}$. Действительно, из взаимной сопряженности матриц $\left(\partial \Omega_{a} / \partial S_{i}\right)$ и $\left(\partial \omega_{i} / \partial L_{a}\right)$ (теорема I.2) следует, что вектор $\left(d S_{i}\right)$, даюший нулевые углы дефекта вокруг ребер, должен быть ортогонален образу матрицы $\left(\partial \omega_{i} / \partial L_{a}\right)$, т.е. всем ее столбцам. Рассматривая столбец, соответствуюший ребру $a=A B$, и применяя формулу (I.5) и формулы, получаемые из нее заменами $C \leftrightarrow D, C \leftrightarrow E$ и $C \leftrightarrow F$, получим:

$\frac{\partial \omega_{A B C}}{\partial L_{A B}}: \frac{\partial \omega_{A B D}}{\partial L_{A B}}: \frac{\partial \omega_{A B E}}{\partial L_{A B}}: \frac{\partial \omega_{A B F}}{\partial L_{A B}}=V_{-\widehat{C}} S_{A B C}: V_{\widehat{D}} S_{A B D}: V_{-\widehat{E}} S_{A B E}: V_{\widehat{F}} S_{A B F}$.

Это сразу приводит к линейной зависимости (11), и остается заметить, что между нашими четырьмя $d S$ сушествует только одна зависимость.

Тот факт, что равенство (11), с одной стороны, выполнено для дифференциалов плошадей, порожденных девиациями ребра $A B$, а с другой - эквивалентно условию $d \Omega_{A B}=0$, показывает, что мы доказали следуюшую лемму.

Лемма 2. Если ребро АВ является общим для ровно четырех 4-симплексов, то порождаемые девиачиями $d \vec{v}_{A B}$ бесконечно малье изменения площадей порождают в свою очередь угол дефекта $d \Omega_{A B}=0$.

Мы хотим доказать, что девиации всех ребер комплекса порождают такие $d S_{i}$, что все $d \Omega_{a}=0$. Докажем это сначала для комплекса, представляющего собой триангуляцию сферы $S^{4}$, содержашую шесть вершин $A, \ldots, F$ и шесть 4 -симплексов $\hat{A}, \ldots, \widehat{F}$. 
В таком комплексе, во-первых, каждое ребро является общим для ровно четырех 4-симплексов. Во-вторых, все $d \Omega_{a}$ пропорциональны (ранг матришы $\left(\partial \Omega_{a} / \partial S_{i}\right)$ равен 1$)$. Поэтому лемма 2 сразу дает нужньй результат.

Если теперь рассмотреть произвольный комплекс, в котором ребро $A B$ является общим для ровно четырех 4-симплексов, то девиации всех ребер снова дают $d \Omega_{A B}=0$, ибо $d \Omega_{A B}$ может в принципе зависеть лишь от девиаций ребер, принадлежаших четырем ближним симплексам, и эта зависимость такая же, как в предыдушем случае триангуляции $S^{4}$.

Остается рассмотреть случай, когда вокруг ребра $A B$ расположено больше четырех 4-симплексов. Мы всегда можем считать, что наша триангуляция многообразия получена с помошью движений Пахнера, т.е. перестроек $3 \rightarrow 3,2 \leftrightarrow 4$ и $1 \leftrightarrow 5$, из другой триангуляции, в которой не было ребра $A B$. Это ребро появилось в результате перестройки $2 \rightarrow 4$ или $1 \rightarrow 5$ и, таким образом, его вначале окружали четыре 4 -симплекса, так что все производные $\left(\partial \Omega_{A B} / \partial \vec{v}_{a}\right)$ были равны нулю. Далее в окрестности ребра $A B$ могли быть произведены еше перестройки разных типов, но каждую из них можно интерпретировать следуюшим образом: кластер из нескольких 4-симплексов, находившихся в симплициальном комплексе, заменяется на другой кластер, причем два эти кластера вместе образуют триангуляцию сферы $S^{4}$ на шесть 4-симплексов (описанную выше). Можно сказать, что к комплексу добавляется шесть новых 4-симплексов, причем часть из них сокращается с уже сушествовавшими, так как их ориентации противоположны. Вместе с шестью добавляемыми симплексами (ориентации которых согласовань между собой при любой перестройке) ко всем углам дефекта в комплексе добавляются углы дефекта в $S^{4}$. Так как и там, и там все производные $\partial \Omega_{A B} / \partial \vec{v}_{a}$ равны нулю, они остаются нулевыми и после перестройки.

Таким образом, теорема 2 доказана.

\section{6. ПЕРЕСТРОЙКИ $2 \leftrightarrow 4$ И ИНВАРИАНТ ПЕРЕСТРОЕК $3 \rightarrow 3$}

В статье I мы построили инвариант (I.23) только для перестроек $3 \rightarrow 3$ в виде произведения двух дифференциальных форм (действуюших на внешних степенях двух разных линейных пространств). С точки зрения наших комплексов (6) и (7), мы изучали только отображение $\left(\partial \Omega_{a} / \partial S_{i}\right)$ и сопряженное ему $\left(\partial \omega_{i} / \partial L_{a}\right)$. Выражение (I.23) не может быть инвариантным относительно перестроек $2 \leftrightarrow 4$, так как при этом изменяется степень формы $\wedge d S$ - добавляется три новых дифференциала. Покажем, однако, что в предположении точности последовательности (6) в члене $\left(d S_{i}\right)$ поведение выражения (I.23) при перестройках $2 \leftrightarrow 4$ описывается очень просто.

Ниже мы будем рассматривать переход от двух 4-симплексов к четырем, т.е. перестройку $2 \rightarrow 4$. Все наши рассуждения и формулы допускают обрашение на случай перестройки $4 \rightarrow 2$.

Итак, пусть два смежных 4-симплекса $A C D E F=\widehat{B}$ и $B C D F E=-\hat{A}$ заменяются на четыре: $A B C D E=\widehat{F}, A B C F D=-\widehat{E}, A B C E F=\widehat{D}$ и $A B D F E=-\widehat{C}$. При этом в комплексе возникает новое ребро $A B$ и четыре двумерных грани, содержаших это ребро. Рассуждения, аналогичные приведенным в разделе 3 работы [2], показывают, что

2 Теоретическая и математическая физика, т. 133, № 1, 2002 г. 
ранг матрицы $\left(\partial \omega_{i} / \partial L_{a}\right)$ при этом возрастает на единицу, и если мы добавим ребро $A B$ к множеству ребер $\mathcal{C}$ (см. работу I, раздел 5 ) и, например, грань $A B F$ к множеству граней $\mathcal{D}\left(\right.$ см. там же), то определитель матришы $\mathcal{B}={ }_{\mathcal{D}}\left(\partial \omega_{i} / \partial L_{a}\right) \mathcal{C}$ при нашей перестройке умножается на $\pm \partial \omega_{A B F} / \partial L_{A B}$ :

$$
\operatorname{det} \mathcal{B} \underset{2 \rightarrow 4}{\longrightarrow} \pm \frac{\partial \omega_{A B F}}{\partial L_{A B}} \operatorname{det} \mathcal{B}
$$

Эту частную производную можно получить, сопоставляя формулу (I.5) для аналогичной производной $\partial \omega_{A B C} / \partial L_{A B}$ с формулой (13):

$$
\frac{\partial \omega_{A B F}}{\partial L_{A B}}=\frac{S_{A B F}}{24} \frac{V_{\hat{A}} V_{\widehat{B}}}{V_{\widehat{C}} V_{\widehat{D}} V_{\widehat{E}}} .
$$

Используя формулы (14) и (15), находим, что инвариант (I.23) умножается при перестройке $2 \rightarrow 4$ на величину

$$
\pm 3 \frac{d\left(S_{A B C}^{2}\right) \wedge d\left(S_{A B D}^{2}\right) \wedge d\left(S_{A B E}^{2}\right)}{V_{\widehat{F}}}
$$

(разумеется, новые дифференциалы плошадей умножаются внешним образом на уже имевшиеся).

Вернемся к нашему предположению о точности последовательности $(6)$ в члене $\left(d S_{i}\right)$. Нам понадобится включение, обратное доказанному в теореме 2, т.е.

$$
\operatorname{Ker}\left(\frac{\partial \Omega_{a}}{\partial S_{i}}\right) \subset \operatorname{Im}\left(\frac{\partial S_{i}}{\partial \vec{v}_{a}}\right) .
$$

Оно означает, что любой вектор-столбец $\left(d S_{i}\right)$, приводящий к нулевым $d \Omega_{a}$, может быть получен из девиаций $d \vec{v}_{a}$. Из этого следует, что и все внешнее произведение $\wedge d S$ в инварианте (І.23) может быть выражено через девиации ребер.

Включение $(17)$ сохраняется при перестройке $2 \rightarrow 4$ : всегда можно подобрать три компоненты новой девиации $d \vec{v}_{A B}$ так, чтобы получить любые значения трех новых независимых (при условии $d \Omega_{a}=0$ ) дифференциалов плошадей. Новые $d S$ (входяшие в формулу (16)) зависят не только от $d \vec{v}_{A B}$, но и от других девиаций, но зависимость от этих последних не играет роли при взятии внешнего произведения $\wedge d S$ в (I.23), ибо $\wedge d S$ всегда имеет максимальную степень, совпадаюшую с наибольшим количеством линейно независимых $d S$, которые можно получить из девиаций ребер. Поэтому внешнее произведение (16) можно переписать с помощью формулы (10). Отсюда следует

ТЕОРема 3. В предположении, что выполнено включение (17), инвариант (I.23) перестроек $3 \rightarrow 3$ умножсается при перестройке $2 \rightarrow 4$ на величину

$$
\pm 72 L_{A B}^{5 / 2} d v_{x} \wedge d v_{y} \wedge d v_{z}
$$

где $А В$ - добавляемое ребро, $а d v_{x}, d v_{y} u d v_{z}$ - компоненть его девиации. 


\section{7. ОБСУЖДЕНИЕ РУЗУЛЬТАТОВ}

Теорема 3 показывает, что инвариант перестроек $3 \rightarrow 3$, введенный в работе [1], в действительности меняется весьма просто и при перестройках $2 \leftrightarrow 4$, во всяком случае, в предположении включения (17). Можно проверить, что это включение выполнено для сферы $S^{4}$. Теорема 3 показывает также обоснованность рассмотрения как минимум части последовательности (6) от члена $\left(d \Omega_{a}\right)$ до $\left(d \vec{v}_{a}\right)$.

В следующей, третьей, работе этой серии планируется построить линейное пространство девиаций вериин $d \sigma$ и его отображение в пространство девиаций ребер $d \vec{v}$. Это отображение будет тесно связано с перестройками $1 \leftrightarrow 5$. Таким образом, мы сможем учесть все движения Пахнера и построить полньй инвариант четырехмерного кусочно-линейного многообразия с помошью кручения последовательности (6). Для этого требуется, чтобы она была ациклическим комплексом - мы убедимся, что это именно так, в частности, для сферы $S^{4}$. Мы также докажем, что само свойство точности сохраняется при движениях Пахнера.

Заметим, что изучение последовательности (6) выглядит очень интересным, даже если она не окажется точной для какого-либо многообразия. По некоторым соображениям (связанным с теоремой стабилизации Уолла), таковым может оказаться произведение двумерных сфер $S^{2} \times S^{2}$.

Попутно мы перевели на язык кручения ашиклических комплексов соответствуюшие конструкции из работ [2], [3] и тем самым прояснили их алгебраическую сушность. Схематично говоря, в наших работах участвуют, во-первых, решения уравнения пентагона или его обобшений (для размерностей больше 3) и, во-вторых, ациклические комплексы. В этой связи было бы весьма интересно построить аналогичные комплексы, соответствуюшие $S L(2)$-решению уравнения пентагона из работы [5] и его аналогам для большего числа измерений.

Самым же интригуюшим представляется вопрос, пределом каких квантовых объектов являются наши конструкции, имеюшие явно квазиклассический вид.

Благодарности. Работа выполнена при финансовой поддержке Российского фонда фундаментальных исследований, грант № 01-01-00059.

\section{Список литературы}

[1] И. Г. Корепанов. ТМФ. 2001. Т. 131. № 3. С. 377-388.

[2] I. G. Korepanov. J. Nonlinear Math. Phys. 2001. V. 8. № 2. P. 196-210.

[3] I. G. Korepanov, E. V. Martyushev. J. Nonlinear Math. Phys. 2002. V. 9. № 1. P. 86-98.

[4] Дж. Милнор. Кручение Уайтхеда. Математика (сб. переводов). 1967. Т. 11. № 1. С. 3-42.

[5] И. Г. Корепанов, Е. В. Мартюшев. ТМФ. 2001. Т. 129. № 1. С. 14-19.

Поступила в редакцию 4.II.2002 г. 